\title{
NONLINEAR MULTIPLE MODEL PREDICTIVE CONTROL IN A FED-BATCH REACTOR
}

\author{
Ana Isabel González Santos* Tor Arne Johansen ** \\ Jesús Ma. Zamarreño Cosme ${ }^{* * *}$ \\ * Univ. de Valladolid.Dpto.Ingeniería de Sistemas y Automática. \\ Facultad de Ciencias.Spain.ana@autom.uva.es \\ ** Norwegian University of Science and Technology. Department \\ of Engineering Cybernetics. $N$-74491 Trondheim. \\ Norway.Tor.Arne.Johansen@itk.ntnu.no \\ *** Univ. de Valladolid.Dpto.Ingeniería de Sistemas y \\ Automática. Facultad de Ciencias. Spain.jm@autom.uva.es
}

\begin{abstract}
In this work we study the use of nonlinear model predictive control for the control of fed-batch processes. The main idea is to use composite nonlinear models consisting of multiple linear models that are identified and interpolated. The approach is illustrated by a simulation study of a fed-batch process for the synthesis of hexyl monoester maleic acid. Copyright (c) 2000 IFAC
\end{abstract}

Keywords: Batch control, Predictive control, Identification, Nonlinear systems, Optimization problems.

\section{INTRODUCTION}

In this work we study the use of nonlinear model predictive control (NMPC) for the control of batch and fed-batch processes. Such processes are common in industries where the linear model predictive control (LMPC) strategy is well accepted. However, the highly nonlinear nature of most batch and fed-batch processes suggest that performance will be lost when using LMPC rather than NMPC.

Industrial implementations of LMPC rely on most cases on experimental models that are derived using designed experiments and systems identification. For the application of NMPC, the development of a nonlinear prediction model is a major bottleneck, in addition to the design of nonlinear state estimators and implementation of reliable nonlinear programming algorithms for the real-time optimization. Any practical NMPC implementation must resolve the above mentioned problems. In this work we focus our attention to
NMPC of batch processes, and like (Foss et al. 1995), (Townsend et al. 1998), (Trierwiler and Secchi 1998), (González Santos et al. 1999), (Mollov et al. 1998), (Roubos et al. 1998) we investigate the use of a composite global model based on multiple local models as an underlying model representation in cases when a global nonlinear model is not readily available. Such models offer the advantage that they are still transparent in the sense that their structure posses a useful interpretation for both model development and validation.

\section{NONLINEAR MPC OF BATCH PROCESSES}

Optimization problems in batch or fed batch processes are dynamic problems involving highly nonlinear process models. This problem can be formulated, in general form, as:

$$
\min _{u, t_{f}} J\left(x\left(t_{f}\right), u, t_{f}\right)
$$


subject to

$$
\begin{gathered}
\dot{x}=f(x, u, t), x\left(t_{o}\right)=x_{o} \\
h(x, u, t) \leq 0
\end{gathered}
$$

where $x$ are states variables, $u$ are control inputs defined on the time interval $t_{o}$ to $t_{f}$, and $h$ represents state and input constraints.

The objective is operate the process maximising an economic objective function $J . J$ is a function of the states at the final time $t_{f}$ and the final time itself. The resulting problem is a non-linear dynamic optimization problem, and (Rupppen et al. 1995), (Luus and Okongwu 1999), (Loeblin et al. 1999) and (Fournier et al. 1999) describe similar formulations. The optimization problem is defined on a batch time where $t_{f}$ defines the end of the batch, and this variable could be optimised too. At each sampling time, the optimizer finds a future control input trajectory by optimizing over a prediction horizon that is an interval between actual time $t$, and the final batch time $t_{f}$.

The first sample of this input trajectory is applied to the plant, and the optimization is repeated at the next sample using the new states of the plant. Often, some states can not be measured and in this case it is necessary to introduce state estimators in this formulation, e.g. (Laksmanan and Arkun 1999).

\section{MULTIPLE LOCAL MODELS}

This section reviews a technique for developing composite non-linear models based on multiple local models, (Murray-Smith and Johansen 1997). Consider the problem of developing a state-space model of the form:

$$
\begin{gathered}
\dot{x}=f(x, u, t) \\
y=g(x, t)
\end{gathered}
$$

where $x$ is the state vector, $u$ is the control input vector, and $y$ is the measurement vector. When the system operates within a small operating regime, a simple (possibly linear) local model structure:

$$
\begin{gathered}
\dot{x}=f_{i}\left(x, u, p_{i}, t\right) \\
y=g\left(x, p_{i}, t\right)
\end{gathered}
$$

parameterized with the vector $p_{i}$ will describe the system dynamics, provided the nonlinearities are smooth. The local model structure will be valid within this particular operating regime, and more or less invalid outside this regime. The choice of which variables, $z$, to use to characterize the operating regimes will be highly problem dependent. Typically, $z$ will contain elements of the state and input vectors, i.e. given by a function $z=H(x, u)$, but can also contain other model variables. The full range of operation is the set of operating points $Z$. An operating regime is defined as a subset $Z_{i}$ where the local model structure above is an adequate description of the system.

Next, assume that for the local model structure above there exists a local model validity function $\rho_{i}(z) \geq 0$ that is designed such that its value is close to one for operating points $z$ where the local model structure above is a good description of the system, and close to zero otherwise. If the system's operating range $Z$ is decomposed into $N$ operating regimes, $Z_{1}, \ldots, Z_{N}$, and local model structures and local model validity functions for each operating regime are developed, then the following interpolation gives a global model structure:

$$
\begin{gathered}
\dot{x}=\sum_{i=1}^{N} f_{i}\left(x, u, p_{i}, t\right) w_{i}(z) \\
y=\sum_{i=1}^{N} g\left(x, p_{i}, t\right) w_{i}(z) \\
w_{i}(z)=\frac{\rho_{i}(z)}{\sum_{j=1}^{N} \rho_{j}(z)}
\end{gathered}
$$

The interpolation function $w_{i}$ is a normalization of the model validity function, and has the property:

$$
\sum_{i=1}^{N} w_{i}(z)=1
$$

for all $z$. To guarantee a complete global model, it must be assumed that at any operating point $\mathrm{z}$, at least one local model validity function is nonzero. With this framework, the modeling problem consists of the following major tasks:

(1) First, decompose the system's operating range into a number of operating regimes that completely cover the interesting range of operation. Such a decomposition can often be found by using an elementary understanding of the mechanisms in the system.

(2) Second, for each operating regime, a local model structure must be developed. In addition, local model validity functions must be designed. However, this is usually a quite straightforward task when the decomposition into regimes has been accomplished.

(3) Third, the unknown parameters $p_{1}, \ldots, p_{N}$ must be identified. If the local model structures are linearly parameterized, the local model validity function do not contain unknown parameters. Standard system identification tools can be applied. Since the model is nonlinear it is particularly important with 
informative data that covers all operating regimes with local models that contain unknown parameters.

\section{SIMULATION EXAMPLE}

Multiple local models that describe the non-linear dynamics are obtained and validated with ORBIT, a MATLAB based tool for operating regime based modelling and identification, (Johansen and Foss 1998), in a realistic simulation study.

\subsection{System description}

The simulated true system model describe a synthesis of hexyl monoester maleic acid according to maleic anhydride and hexanol reaction. In fedbatch operation, reactant A (maleic anhydride) is first melted; then reactant B (hexanol) is added at a regulated rate so that the heat generated is matched by the cooling capacity. The following state-space model is used to simulate the true system (Chang and Hseih 1995):

$$
\begin{gathered}
\dot{C}_{a}=-\frac{C_{a} U_{1}}{V_{r}}-k C_{a} C_{b} \\
\dot{C}_{b}=\frac{\left(C_{b l}-C_{b}\right) U_{1}}{V_{r}}-k C_{a} C_{b} \\
\dot{T}=-\frac{\Delta H}{\rho C_{p}} k C_{a} C_{b}-(T-327) \frac{U_{1}}{V_{r}} \\
-U_{2}(T-327) \\
\dot{V}_{r}=U_{1} \\
k=k_{o} \exp (-12628 / T)
\end{gathered}
$$

Input variables are reactive flow rate $U_{1}$ and cooling fluid flow rate $U_{2}$. State variables are reagent A (maleic anhydride) and reagent B (hexanol) concentrations $C_{a}, C_{b}$, reactor temperature $T_{r}$ and reactor volume $V_{r}$. Feed flow of reagent $\mathrm{B}$ has concentration $C_{b l}$. Figure 1 shows three system trajectories corresponding to different constant inputs, when the batch time is equal to $t_{f}=6700$ s. In the upper plot, $U_{1}=1.0 \cdot 10^{-5}$ and $U_{2}=1.0$. $10^{-5}$, while in the middle plot, $U_{1}=0.355 \cdot 10^{-3}$ and $U_{2}=2.0 \cdot 10^{-5}$, and in the lower plot $U_{1}=1.0$. $10^{-2}$ and $U_{2}=253 \cdot 10^{-6}$. At higher feed rates, greater than $U_{1}=0.355 \cdot 10^{-3} \mathrm{~m}^{3} / \mathrm{s}$, the maximum allowable temperature $(373 K)$ is exceeded. Model parameters and nominal initial state of fed-batch reactor appear in Table 1.
Table 1. Model parameters

\begin{tabular}{lll}
\hline Parameter & Value & Units \\
\hline$C_{b l}$ & 9.7 & $\mathrm{kmol} / \mathrm{m}^{3}$ \\
$k_{o}$ & $1.37 * 10^{12}$ & $\mathrm{~m}^{3} / \mathrm{kmols}$ \\
$\frac{\Delta H}{\rho C_{p}}$ & 16.92 & $\mathrm{~m}^{3} \mathrm{~K} / \mathrm{kmol}$ \\
$C_{a o}$ & 10.1 & $\mathrm{kmol} / \mathrm{m}^{3}$ \\
$C_{b o}$ & 0.0 & $\mathrm{kmol} / \mathrm{m}^{3}$ \\
$T_{o}$ & 328 & $\mathrm{~K}$ \\
$V_{r o}$ & 2.2 & $\mathrm{~m}^{3}$ \\
$U_{1, \min }$ & 0 & $\mathrm{~m}^{3} / \mathrm{s}$ \\
$U_{1, \max }$ & 0.01 & $\mathrm{~m}^{3} / \mathrm{s}$ \\
$U_{2, \min }$ & 0 & $\mathrm{~s}^{-1}$ \\
$U_{2, \max }$ & 0.000253 & $\mathrm{~s}^{-1}$ \\
\hline
\end{tabular}
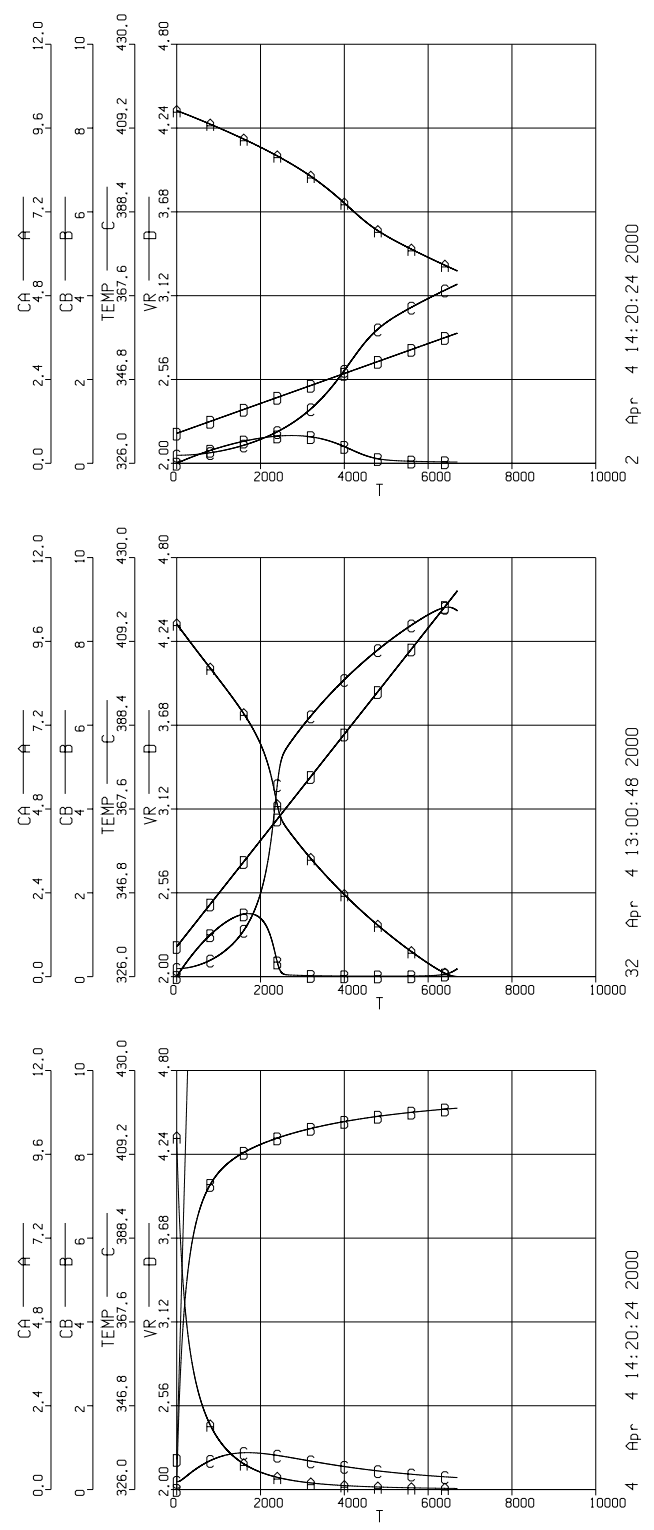

Fig. 1. Typical state trajectories.

\subsection{Modeling and Identification}

All the local models are chosen to have the same linear structure:

$$
x(t+1)=a_{i}+A_{i} x(t)+B_{i} u(t)
$$




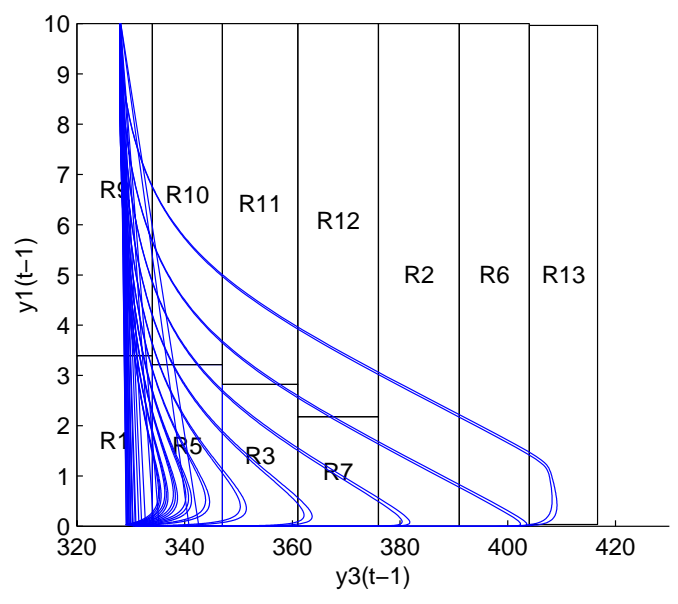

Fig. 2. Operating regimes.

where $x=\left(C_{a}, C_{b}, T_{r}, V_{r}\right), u=\left(U_{1}, U_{2}\right), a_{i}$ is a vector of unknown parameters and $A_{i}$ and $B_{i}$ are system matrices of unknown parameters. For different initial conditions and input trajectories a number of representative batch simulations realized and identification data were generated.

Using ORBIT (Johansen and Foss 1998) we identified and validated a composite model consisting of 11 operating regimes with local linear models within each regime. The operating regimes are characterized using the variables $T_{r}$ and $C_{a}$ as shown in Figure 2.

Notice that the equation for the volume (15) was not identified since it was known a priori.

\subsection{Model Predictive Control}

The objective is to obtain a final conversion of product $\mathrm{B}$ concentration to product A concentration in $99 \%$. The optimization problem is formulated as:

$$
\min _{U_{1}(t), U_{2}(t)} J=\left(\operatorname{conv}\left(t_{f}\right)-99 \%\right)^{2}
$$

subject to the model equations (12),(13),(14),(15) and (16) and the additional constraints:

$$
\begin{gathered}
U_{1}(t) \in[0.0,0.01] \\
U_{2}(t) \in[0.0,0.000253] \\
T \leq 373 \mathrm{~K} \\
V_{r} \leq 4.7 \mathrm{~m}^{3}
\end{gathered}
$$

Conversion is calculated as

$$
\operatorname{conv}\left(t_{f}\right)=1-\frac{V_{r}\left(t_{f}\right) C_{a}\left(t_{f}\right)}{V_{r}\left(t_{o}\right) C_{a}\left(t_{o}\right)}
$$

where $t_{o}$ is the start time of the batch.

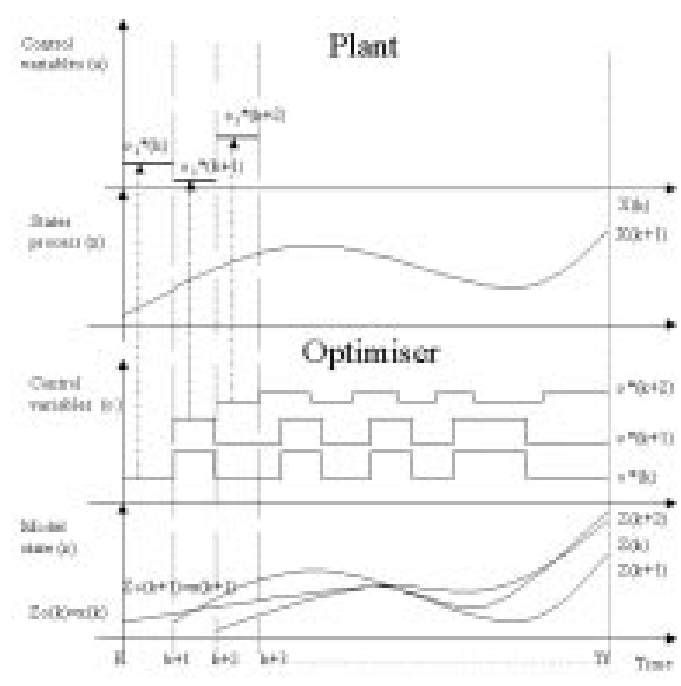

Fig. 3. Nonlinear optimization problem strategies.

The input constraints (19), (20) and the volume constraint (22) represents physical limitations, while (21) is an upper bound on the allowed temperature.

The trajectories are optimized from time $t$ to the batch end time $t_{f}$, which nominally equals 6700 seconds. The sampling interval of the controller is $\Delta T=100$ seconds.

At each sampling time, states are measured and above optimization problem was resolved in order to determine the optimum inputs to reach the objective. Figure 3 illustrates this procedure. The first value of the input trajectories were applied to the plant and the procedure is repeated at the next sample.

We consider two cases:

- NMPC using the perfect model in the optimization for prediction.

- MMPC (multi-model predictive control) where the identified composite model is used for prediction in the optimization.

In both cases, the criterion (18) is minimized using e04jbc NAG quasi-Newton algorithm, (NAG.Ltd 1996).

\subsection{Results and discussion}

The achieved cost (18) are summarized in Table 2 for three different initial conditions. Figures $4,6,8$ and 10 show simulation results with NMPC. Figures 5, 7, 9 and 11 show simulations results with MMPC. These figures correspond to first simulation.

Table 2 also shows the final batch time. Results with MMPC show good results. The stop condition is when the volume $V_{r}$ reaches the maximum capacity of reactor, due to, final time is different for every simulation. Constraint (21) was realized 
Table 2. Achieved cost

\begin{tabular}{lllll}
\hline Exp & NMPC & $t_{f}$ & MMPC & $t_{f}$ \\
\hline 1 & 0.9998 & 6087.5 & 0.9915 & 2120 \\
2 & 0.9909 & 4621.1 & 0.9828 & 2910 \\
3 & 0.9853 & 3730.1 & 0.9710 & 4210 \\
\hline
\end{tabular}

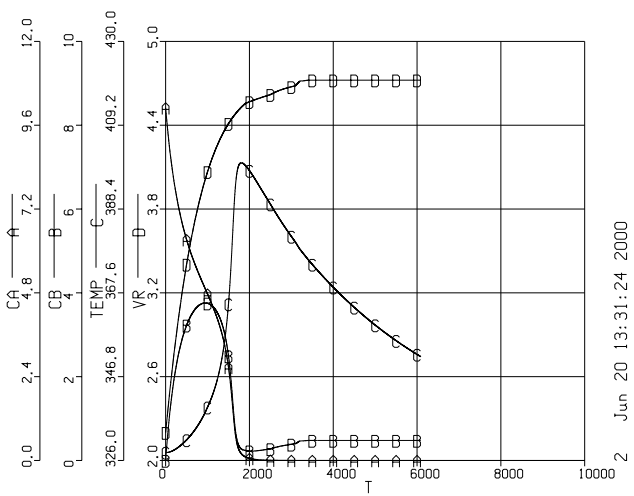

Fig. 4. Concentration A and B products, temperature and volumen with NMPC Results

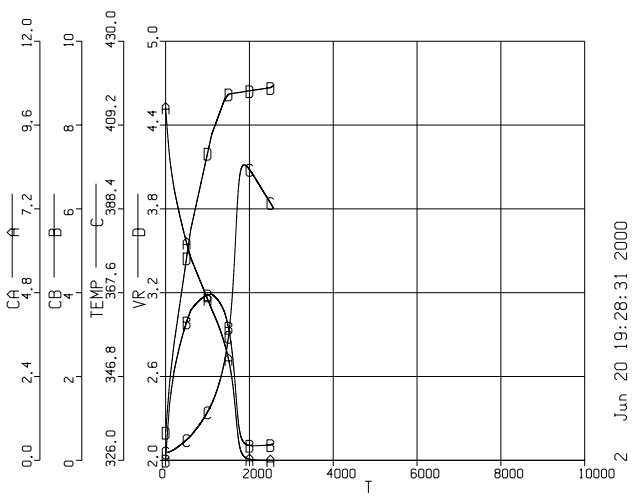

Fig. 5. Concentration A and B products, temperature and volumen with MMPC Results

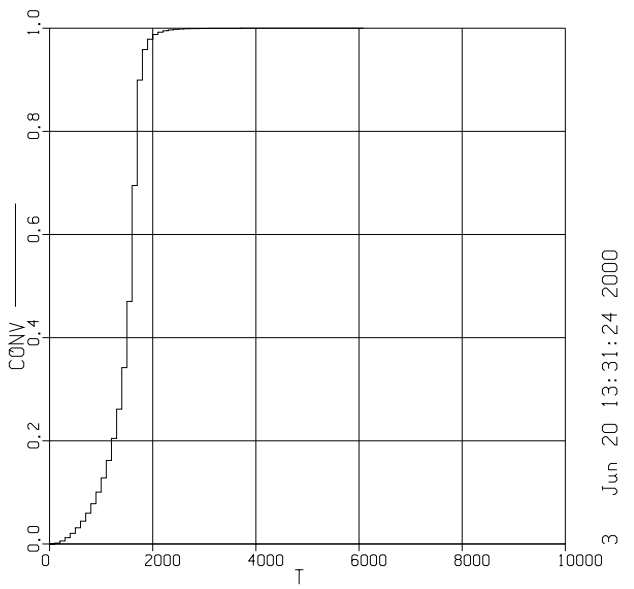

Fig. 6. Conversion achieved with NMPC Results

as a soft penalty function. The upper temperature of this reaction is less critical than for other reactions. This reason explains why violation in upper limit was permitted as show figures 4 and 5 . Initial conditions are the same, $U_{1}$ is $0.008 \mathrm{~m}^{3} / \mathrm{s}$ and

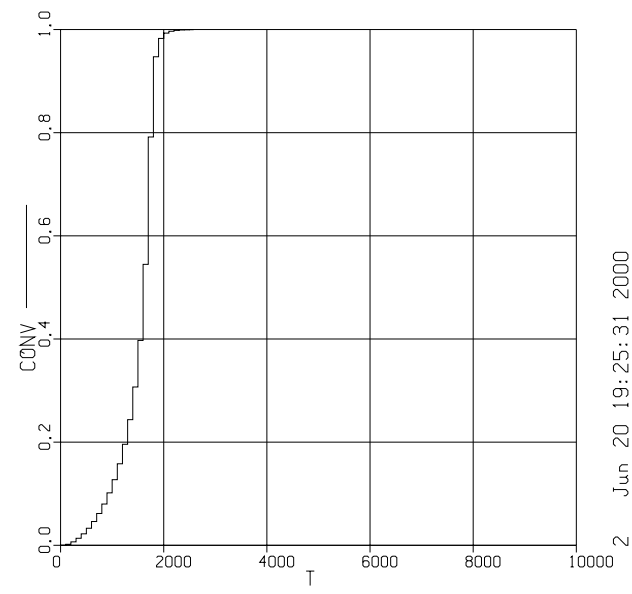

Fig. 7. Conversion achieved with MMPC Results

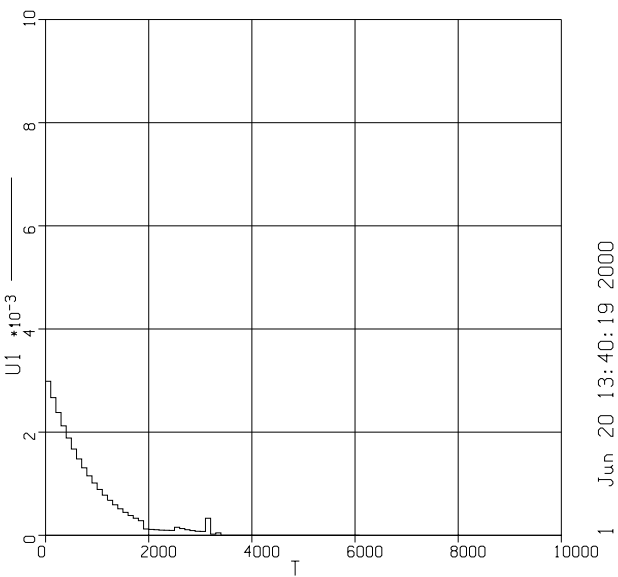

Fig. 8. Reactive flow input with NMPC Results

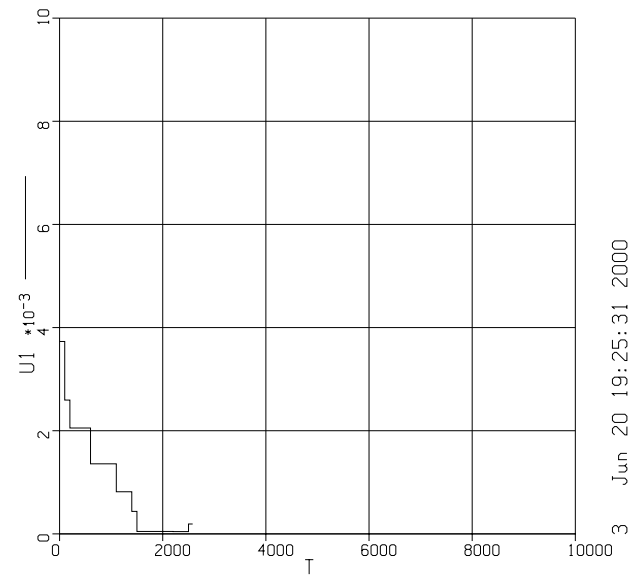

Fig. 9. Reactive flow input with MMPC Results

$U_{2}$ is $0.000253 \mathrm{~s}^{-1}$ although variables are changed for the controller in first sampling time as show figures 8, 9, 10 and 11 . 


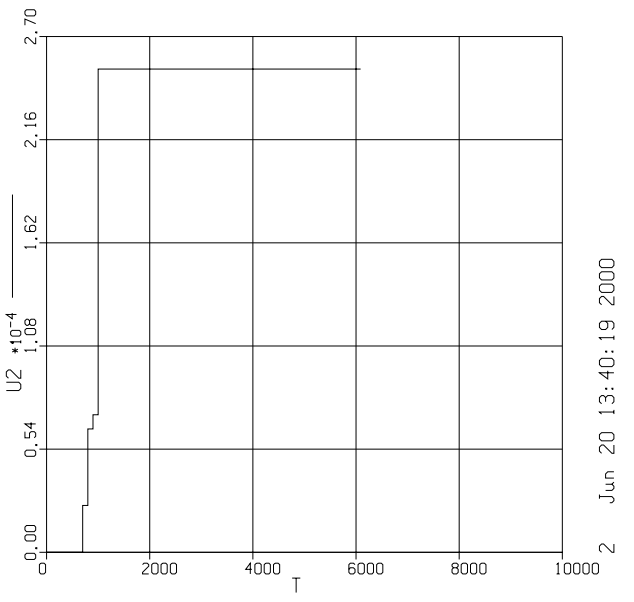

Fig. 10. Cold flow input with NMPC Results

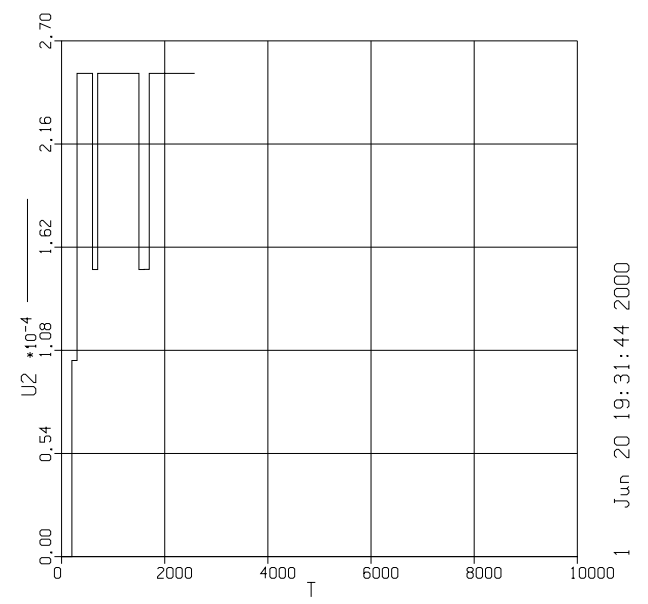

Fig. 11. Cold flow input with MMPC Results

\section{CONCLUSIONS}

This investigation showed that the operating regime based modeling framework can be used as a means for modelling batch and fed-batch processes from experimental data. Simulation experiments show that multiple model predictive control might be a useful alternative in these processes.

Modeling and identification procedure suggest that critical part is characteristic variable selection and regimes splitting. Estimation aspect is obviously important from a practical point view, it has not been considered here because we supposed all states measured.

\section{REFERENCES}

Chang, Jyh-Shyong and Wen-Yen Hseih (1995). Optimization and control of semibatch reactors.. Ind. Eng. Chem. Res. 33, 545-556.

Foss, B.A, T.A Johansen and A.V Sorensen (1995). Nonlinear predictive control using local models-applied to a batch fermentation process. Control Engineering Practice 3, 389396.

Fournier, F., M.A Latifi and G. Valentin (1999). Methodology of dynamic optimization and optimal control of batch electrochemical reactors.. Chemical Eng. Science 54, 2707-2714.

González Santos, A.I, J.M Zamarreño Cosme and C. Prada Moraga (1999). Predictive Optimizer in a Batch Fermentator. Technical report. Internal report. Engineering Systems and Automatic Departament. Valladolid University. Spain.

Johansen, T.A and B.A Foss (1998). Orbitoperating regime based modelling and identification toolkit. Control Engineering Practice 6, 1277-1286.

Laksmanan, N.M and Y. Arkun (1999). Estimation and model predictive control of nonlinear batch processes using linear parameter varying models. International Journal of Control 9, 61-78.

Loeblin, C., J.D Perkins, B. Srinivasan and D. Bonvin (1999). Economic performance analysis in the design of on line batch optimization systems. Journal of Process Control 9, 61-78.

Luus, R. and O.N Okongwu (1999). Towards practical optimal control of batch reactors. Chemical Eng. Journal. 75, 1-9.

Mollov, S., R. Babuska and H.B Verbruggen (1998). Predictive control by multiple-step linearization of Takagi-Sugeno fuzzy models. In: Proceedings IFAC/IMACS International Conference on Artificial Inteligence in RealTime Control - AIRTC.

Murray-Smith, R. and T.A Johansen (1997). Multiple Model Approaches to Modelling and Control. Taylor\&Francis. London.

NAG.Ltd (1996). Nag-C Libraries. The Numerical Algorithms Group Limited. UK.

Roubos, J.A, R. Babuska, P.M Bruijn and H.B Verbruggen (1998). Predictive control by local linearization of a Takagi-Sugeno fuzzy model. In: Proceedings FUZZ-IEEE'98.

Rupppen, D., C. Benthack and D. Bonvin (1995). Optimization of batch reactor operation under parametric uncertainty-computational aspects. Journal Process Control 5(4), 235-240.

Townsend, S., G. Lightbody, M.D Brown and G.W Irwin (1998). Nonlinear dynamic matrix control using local models. Trans. Inst. Measurement and Control 20, 47-56.

Trierwiler, J.O and A.R Secchi (1998). Exploring the potential of using Multiple Model Approaches in Nonlinear Model Predictive Control. Technical report. Proc. COBEQ '98. Porto Alegre,Brazil. 